\title{
Afatinib for the first-line treatment of EGFR mutation-positive NSCLC in China: a review of clinical data
}

\author{
Hai-Yan Tu' iD \& Yi-Long Wu*,1 iD \\ ${ }^{1}$ Guangdong Lung Cancer Institute, Guangdong Provincial Key Laboratory of Translational Medicine in Lung Cancer, Guangdong \\ Provincial People's Hospital \& Guangdong Academy of Medical Sciences, Guangzhou, 510080, PR China \\ *Author for correspondence: Tel.: +86 208382 7812; Fax: +86 208384 4620; syylwu@live.cn
}

Mutations in the EGFR gene are particularly prevalent among Chinese patients with non-small-cell lung carcinoma. Six EGFR tyrosine kinase inhibitors are approved for the first-line treatment of EGFR mutationpositive non-small-cell lung carcinoma in China, which poses questions about which agent is most suitable for a particular patient. In this article, we review available clinical trial and real-world data with afatinib in Chinese patients. We assess its efficacy and safety in key patient subgroups such as those with uncommon mutations or brain metastases. We also consider possible subsequent therapies following afatinib. Encouragingly, available data suggest that sequential afatinib and osimertinib confer prolonged overall time to failure of almost 4 years in Asian patients, and represents a viable option in this setting.

First draft submitted: 8 April 2020; Accepted for publication: 21 July 2020; Published online: 15 September 2020

Keywords: afatinib • Chinese • EGFR mutation • first-line • NSCLC

In China, lung cancer is the most common malignancy, accounting for $18 \%$ of new diagnoses in 2018 [1]. It is also the leading cause of cancer mortality, taking the lives of over 690,000 people in 2018. Of the lung cancer subtypes, non-small-cell lung cancer (NSCLC) is the most common, and is generally diagnosed at an advanced stage [2]. In recent years, investigation into the molecular basis of NSCLC has led to the identification of a number of targetable molecular aberrations, including mutations in the genes encoding the EGFR and anaplastic lymphoma kinase [3].

Mutations in EGFR are highly prevalent in Chinese patients with NSCLC, being detected in $35-50 \%$ of individuals [4-9]. In contrast, EGFR mutations are found in around 14\% of European and 24\% of North and South American NSCLC patients [10]. In Chinese populations, EGFR mutations are most commonly detected in women, nonsmokers and patients with NSCLC of adenocarcinoma histology $[4,6,7]$. Some geographical variations in incidence rates have been reported, with higher rates of around $46-49 \%$ reported in northern and South-West China [5,7]; conversely, lower detection rates $(\sim 35 \%)$ were reported in the Yunnan province of South-West China [4].

As has been observed worldwide, two subtypes of EGFR mutation are detected in the majority of Chinese patients: the point mutation L858R, and deletions in exon 19 (Del19) [5,7]. Del19 mutations are generally more common than the L858R mutation $[4,5,11,12]$. The uncommon mutations, G719X, L861Q and S768I, have also been detected in Chinese populations, together accounting for approximately $6 \%$ of $E G F R$ mutations in one study in South-West China [7], and 4\% in a population in North China [5]. In a study of 5363 Chinese lung cancer patients, uncommon mutations were seen in $12 \%$ of patients with EGFR mutations. The most frequent uncommon mutations were exon 20 insertions and G719X, and were detected in 31 and 21\% of patients with uncommon mutations, respectively [13].

Currently, the treatment of EGFR mutation-positive $(E G F R \mathrm{~m}+)$ NSCLC in China generally follows the guidelines issued by the Chinese Society of Clinical Oncology (CSCO) [14]. In a joint initiative, the European Society of Medical Oncology (ESMO) and CSCO recently published pan-Asian-adapted clinical practice guidelines for managing patients with metastatic NSCLC [15]. These guidelines are largely similar to those issued by the CSCO, and include a range of recommendations for the first-line treatment of EGFRm + NSCLC. In both guidelines, 
the recommended first-line therapy for patients with advanced EGFRm+ NSCLC is an EGFR tyrosine kinase inhibitor (TKI) [14,15].

Six EGFR TKIs are currently approved in China for the first-line treatment of EGFRm + NSCLC: the reversible first-generation EGFR TKIs, erlotinib, gefitinib and icotinib, which was developed in China [16]; the irreversible ErbB-family blockers, afatinib and dacomitinib; and the third-generation irreversible, wild-type-sparing EGFR TKI, osimertinib [15]. Erlotinib, gefitinib, afatinib and icotinib have all demonstrated improvements in progression-free survival (PFS) versus chemotherapy in Phase III trials of patients with treatment-naive EGFRm + NSCLC [11,17-24]. In subsequent comparative studies, second- and third-generation EGFR TKIs were shown to confer significantly longer PFS versus first-generation EGFR TKIs [25-28]. Of note, in the recent Phase III FLAURA trial, osimertinib demonstrated significantly improved overall survival (OS) compared with gefitinib or erlotinib [29]. Furthermore, in an exploratory analysis, dacomitinib, demonstrated improved OS, as well as PFS, versus gefitinib [27,28]. In the global Phase IIb LUX-Lung 7 study (discussed in detail below), afatinib showed a trend toward improved OS, and significantly improved PFS, versus gefitinib $[25,30]$. While these studies clearly demonstrate the superiority of second- and third-generation TKIs versus first-generation EGFR TKIs, no head-to-head trials have compared outcomes between second- and third-generation TKIs.

As afatinib has been approved in China for only a short time ( $\sim 2$ years) at the time of writing, experience with afatinib in routine clinical practice in China is currently limited. To facilitate a broader understanding of the use of afatinib in our country, we summarize available data on the efficacy and tolerability of afatinib, and the impact of dose adjustments, in Chinese patients. This information is especially relevant given the high prevalence of $E G F R$ mutations among NSCLC patients in China. In addition, we provide a general comparison of the efficacy and safety of the EGFR TKIs in Chinese patients.

\section{Afatinib in Chinese patients: clinical data \\ Efficacy}

Afatinib has undergone a comprehensive clinical development program, including several Phase III studies involving large numbers of Chinese patients. Two pivotal Phase III studies comparing afatinib with platinum-based chemotherapy in patients with advanced EGFRm + NSCLC (including uncommon EGFR mutations) have been conducted: LUX-Lung 3 and LUX-Lung 6. Although no LUX-Lung 3 trial sites were located in mainland China, $72 \%$ of patients were East Asian, drawn from sites in Taiwan, Hong Kong, Thailand, South Korea and Japan [22]. LUX-Lung 6 was conducted in China, Thailand and South Korea, with almost 90\% of afatinib-treated patients from China [23,31].

Afatinib significantly improved PFS versus platinum-based chemotherapy in the overall populations of both Phase III studies [22,23], and in 327 Chinese patients treated in LUX-Lung 6 (Table 1 \& Figure 1A) [31]. In LUXLung 6, Chinese patients with Del19 appeared to derive a slightly greater PFS benefit from afatinib than did those with L858R (hazard ratio [HR]: 0.21 and 0.33, respectively; Figure 1B) [31]. In a preplanned analysis in patients with Del19 mutations, afatinib significantly prolonged OS versus chemotherapy in the overall populations and in Chinese patients (Table $1 \&$ Figure 2) [31,32]. Afatinib was also associated with significantly higher response rates versus chemotherapy, in both the overall and Chinese populations in LUX-Lung 6 [23,31]. In Chinese patients, the objective response rate (ORR) with afatinib was $67 \%$ compared with $24 \%$ in chemotherapy-treated patients $(\mathrm{p}<0.0001)$; further, the disease control rate (DCR) was significantly higher ( $92 \mathrm{vs} 77 \% ; \mathrm{p}=0.0003)$ and median duration of response longer (9.7 vs 4.2 months) with afatinib (Table 1) [31]. Of note, the ORR with afatinib was significantly higher than with platinum chemotherapy in all mutation subgroups examined (L858R, Del19 and others) and was particularly pronounced in Del19-positive patients (ORR: 78 vs 27\%; odds ratio: 9.17 [95\% CI: 4.37-19.26]; $\mathrm{p}<0.0001$ ) [31].

LUX-Lung 7 confirmed the efficacy of first-line afatinib in patients from a range of countries and ethnic backgrounds [25,30]. Of 319 randomized patients, just over half were Asian; of these, 48 (15\% of all patients) were from China. PFS was significantly longer with afatinib compared with gefitinib in the overall population (HR: 0.73 [95\% CI: 0.57-0.95]; $\mathrm{p}=0.017$; Table 1), with no significant differences between Asian and non-Asian patients $($ pinteraction $=0.88$ ) and similar HRs in the two groups (Asian 0.76 [95\% CI: 0.54-1.06]; non-Asian 0.72 [95\% CI: 0.49-1.06]) [25]. In addition, time-to-treatment failure was significantly longer with afatinib than gefitinib (HR: 0.73 [95\% CI: 0.58-0.92]; $\mathrm{p}=0.007$ ), with similar findings in Asians (13.2 vs 11.4 months) and non-Asians (14.1 vs 11.5 months) [25]. A trend toward improved OS with afatinib versus gefitinib was observed in the overall LUX-Lung 7 population (HR: 0.86 [95\% CI: 0.66-1.12]) with no interaction between Asians and non-Asians 
Table 1. Overview of Phase IIb/III studies of afatinib in patients with advanced EGFR mutation-positive NSCLC in which a large proportion of patients were enrolled at sites in China.

\begin{tabular}{|c|c|c|c|c|c|c|c|}
\hline & \multicolumn{4}{|c|}{ LUX-Lung 6} & \multirow{2}{*}{\multicolumn{2}{|c|}{$\begin{array}{l}\text { LUX-Lung-7 } \\
\text { All patients }\end{array}$}} & \multirow{3}{*}{\begin{tabular}{l}
\multicolumn{1}{c}{1200.66} \\
All patients ${ }^{\dagger}$ \\
Afatinib
\end{tabular}} \\
\hline & \multicolumn{2}{|c|}{ All patients } & \multicolumn{2}{|c|}{ Chinese patients } & & & \\
\hline & Afatinib & $\begin{array}{l}\text { Gemcitabine + } \\
\text { cisplatin }\end{array}$ & Afatinib & $\begin{array}{l}\text { Gemcitabine + } \\
\text { cisplatin }\end{array}$ & Afatinib & Gefitinib & \\
\hline Number of patients $(n)$ & 242 & 122 & 217 & 110 & 160 & 159 & 541 \\
\hline \multicolumn{8}{|l|}{ Patient baseline characteristics } \\
\hline Ethnicity/country of origin (\%) & $\begin{array}{l}\text { Chinese: } 90 \\
\text { SE Asian: } 6 \\
\text { South Korean: } \\
5\end{array}$ & $\begin{array}{l}\text { Chinese: } 90 \\
\text { SE Asian: } 8 \\
\text { South Korean: } \\
2\end{array}$ & China: 100 & China: 100 & $\begin{array}{l}\text { Asian: } 59 \\
\text { White: } 30 \\
\text { Other: } 11 \ddagger\end{array}$ & $\begin{array}{l}\text { Asian: } 55 \\
\text { White: } 34 \\
\text { Other: } 11^{\ddagger}\end{array}$ & $\begin{array}{l}\text { China: } 76 \\
\text { Hong Kong: } 5 \\
\text { India: } 9 \\
\text { Singapore: } 4 \\
\text { Taiwan: } 6\end{array}$ \\
\hline Male (\%) & 36 & 32 & 37 & 34 & 43 & 33 & 47 \\
\hline Median age (years; range) & $58(49-65)$ & $58(49-62)$ & $58(30-78)$ & $58(27-75)$ & $63(30-86)$ & $63(32-89)$ & $59(27-82)$ \\
\hline Never smokers (\%) & 75 & 81 & 73 & 84 & 66 & 67 & 69 \\
\hline \multicolumn{8}{|l|}{ Tumor histology (\%) } \\
\hline - Adenocarcinoma & 100 & 100 & 100 & 100 & 99 & 99 & 96 \\
\hline - Mixed/other & 0 & 0 & 0 & 0 & 1 & 1 & 2 \\
\hline - Squamous cell carcinoma & 0 & 0 & 0 & 0 & 0 & 0 & 2 \\
\hline \multicolumn{8}{|l|}{ ECOG PS (\%) } \\
\hline-0 & 20 & 34 & 20 & 36 & 32 & 30 & 18 \\
\hline-1 & 80 & 66 & 80 & 64 & 68 & 70 & 80 \\
\hline-2 & 0 & 0 & 0 & 0 & 0 & 0 & 2 \\
\hline \multicolumn{8}{|l|}{ EGFR mutation type (\%) } \\
\hline - L858R & 38 & 38 & $38^{\S}$ & 38 & 42 & 42 & 40 \\
\hline - Del19 & 51 & 51 & 51 & 50 & 58 & 58 & 48 \\
\hline- L858R + Del19 & NR & NR & NR & NR & 0 & 1 & 0 \\
\hline - Uncommon & 11 & 12 & 11 & 12 & NR & NR & 12 \\
\hline Brain metastases at screening (\%) & NR & NR & NR & $N R$ & 16 & 15 & 19 \\
\hline \multicolumn{8}{|l|}{ Efficacy } \\
\hline Median PFS (months) & 11.0 & 5.6 & 11.0 & 5.6 & 11.0 & 10.9 & 12.1 \\
\hline Median OS (months) & 23.1 & 23.5 & 23.1 & 23.2 & 27.9 & 24.5 & NR \\
\hline$-\mathrm{L} 858 \mathrm{R}$ & 19.6 & 24.3 & 18.7 & 24.5 & 25.0 & 21.2 & \\
\hline - Del19 & 31.4 & 18.4 & 31.6 & 16.3 & 30.7 & 26.4 & \\
\hline \multicolumn{8}{|l|}{ Response } \\
\hline - ORR (\%) & 67 & 23 & 67 & 24 & 72.5 & 56.0 & NR \\
\hline - DCR (\%) & 93 & 76 & 92 & 77 & 91.3 & 87.4 & NR \\
\hline - Median DOR (months) & 9.7 & 4.3 & 9.7 & 4.2 & 10.1 & 8.4 & NR \\
\hline \multicolumn{8}{|l|}{ Safety } \\
\hline Patients with grade $\geq 3 \mathrm{AE}(\%)$ & 36 & 60 & 36 & 57 & 57 & 54 & 45 \\
\hline $\begin{array}{l}\text { Patients undergoing dose } \\
\text { reductions }(\%)\end{array}$ & 28 & NR & 34 & $61 \mathbb{I}$ & 42 & 2 & 29 \\
\hline $\begin{array}{l}\text { Patients requiring treatment } \\
\text { discontinuation due to TRAE (\%) }\end{array}$ & 6 & 40 & 6 & 42 & 6 & 6 & 3 \\
\hline \multicolumn{8}{|l|}{ Most common grade $\geq 3$ TRAEs (\%) } \\
\hline - Rash/acne & 15 & 0 & 16 & 0 & 9 & 3 & 9 \\
\hline - Diarrhea & 5 & 0 & 6 & 0 & 13 & 1 & 11 \\
\hline - Fatigue & $<1$ & $<1$ & 0 & 0 & 6 & 0 & NR \\
\hline - Stomatitis/mucositis & 5 & 0 & 6 & 0 & 4 & 0 & 4 \\
\hline - Neutropenia & $<1$ & 27 & $<1$ & 28 & $<1$ & 0 & $N R$ \\
\hline - Vomiting & 1 & 19 & $<1$ & 19 & 0 & $<1$ & $N R$ \\
\hline - Leucopenia & $<1$ & 15 & $<1$ & 17 & NR & NR & $N R$ \\
\hline - Paronychia & 0 & 0 & NR & NR & 2 & $<1$ & 3 \\
\hline
\end{tabular}

Percentages may not sum to 100 due to rounding.

${ }^{\dagger}$ All patients were TKI-naive; $60 \%$ were treatment naive.

¥Includes 17 patients in each arm who were from France, where ethnic details could not be captured by law.

$\S$ Includes four patients with both L858R and Del19 mutations.

$\mathbb{I}_{39 \%}$ received all courses without interruption.

Data taken from $[23,25,30-33]$

AE: Adverse event; DCR: Disease control rate; DOR: Duration of response; ECOG PS: Eastern Cooperative Oncology Group performance status; NR: Not reported; NSCLC: Non-small-cell lung cancer; ORR: Overall response rate; OS: Overall survival; PFS: Progression-free survival; SE: South-east; TKI: Tyrosine kinase inhibitors. 
(A)

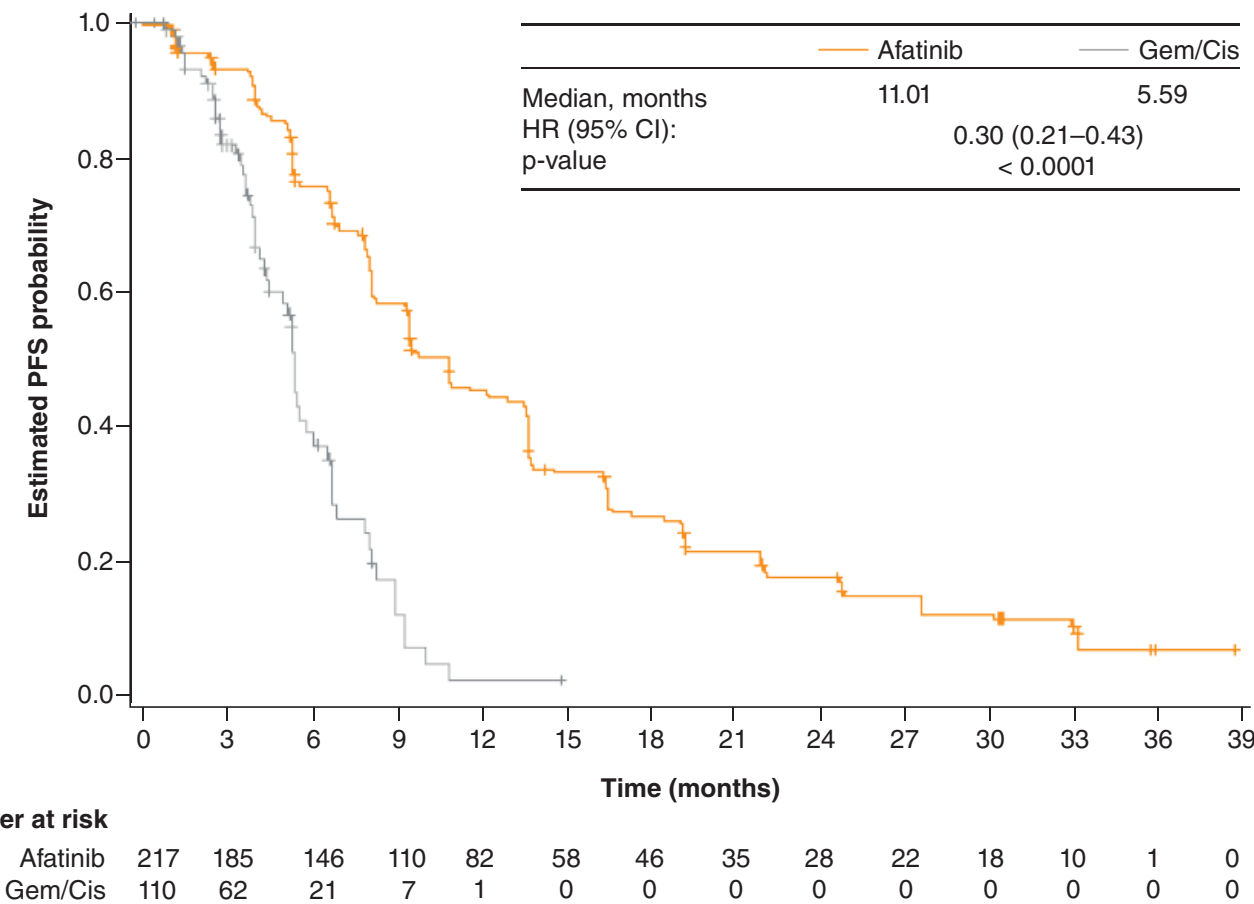

(B)

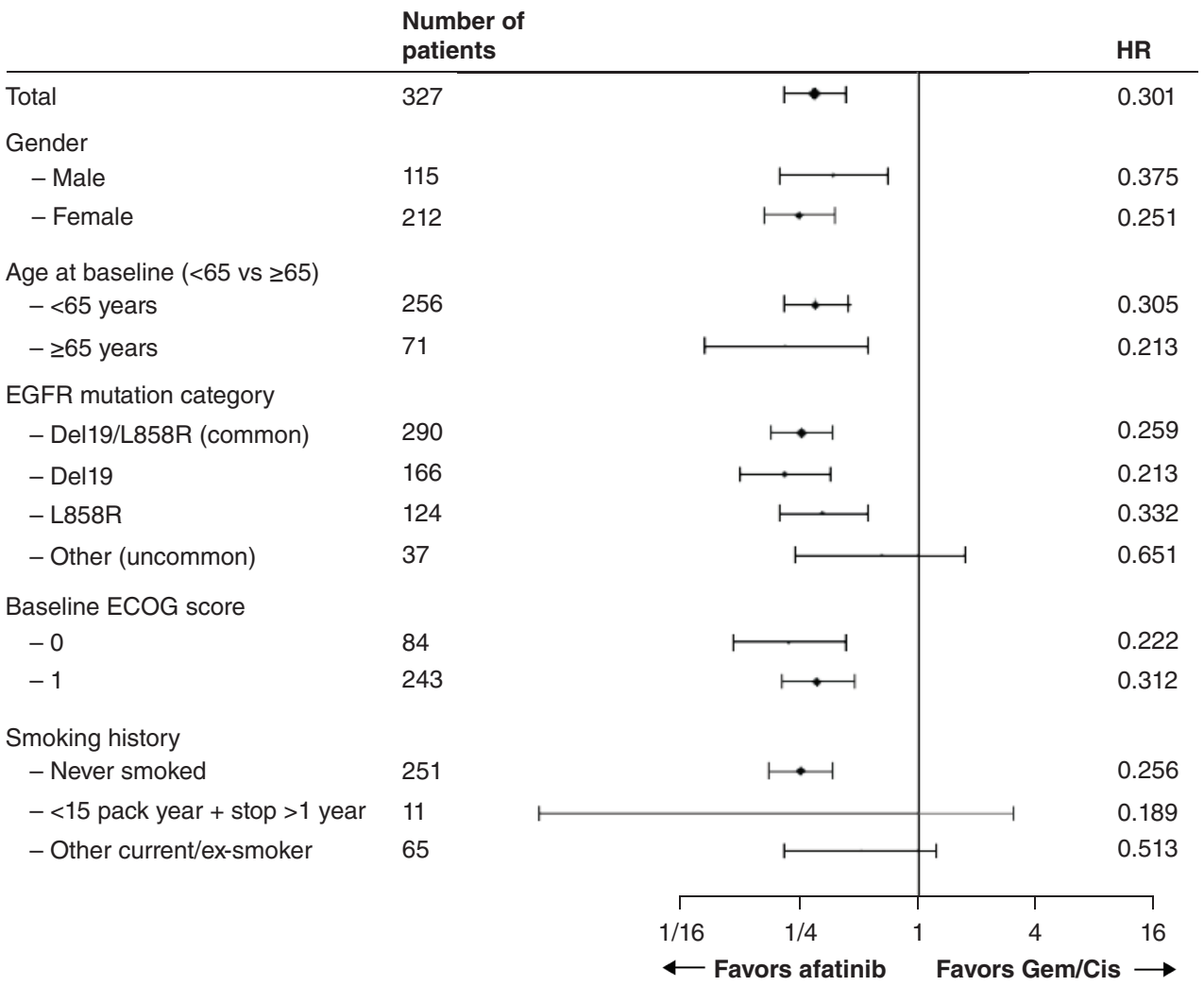

Figure 1. Progression-free survival in Chinese patients treated with afatinib or gemcitabine plus cisplatin in the LUX-Lung 6 study (independent review). (A) Kaplan-Meier curves; (B) forest plot of patient subgroups.

Cis: Cisplatin; ECOG: Eastern Cooperative Oncology Group; Gem: Gemcitabine; HR: Hazard ratio; PFS: Progression-free survival.

Reproduced from [31], with permission from Dove Medical Press Ltd. 
(A)

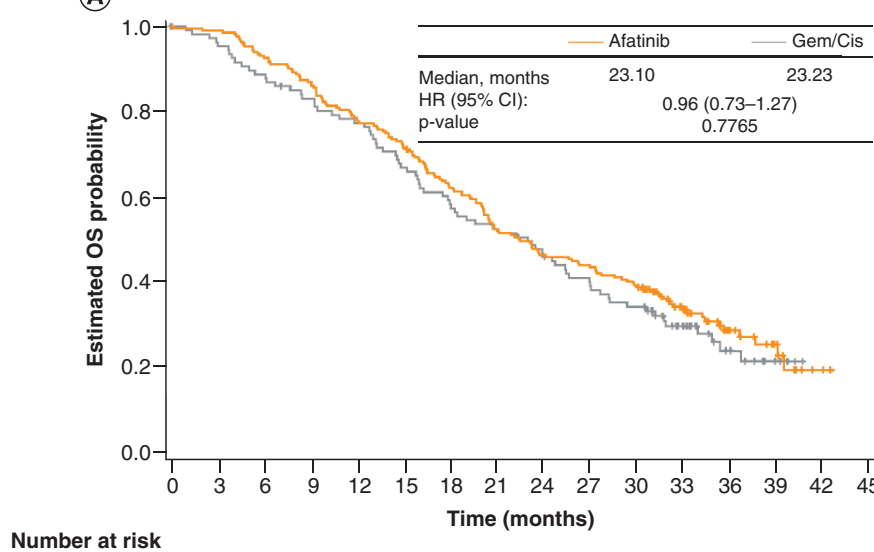

Number at risk

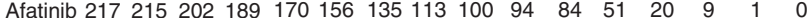
Gem/Cis $110 \begin{array}{lllllllllllllll}103 & 95 & 88 & 82 & 71 & 62 & 56 & 49 & 41 & 36 & 21 & 10 & 4 & 0 & 0\end{array}$
(B)

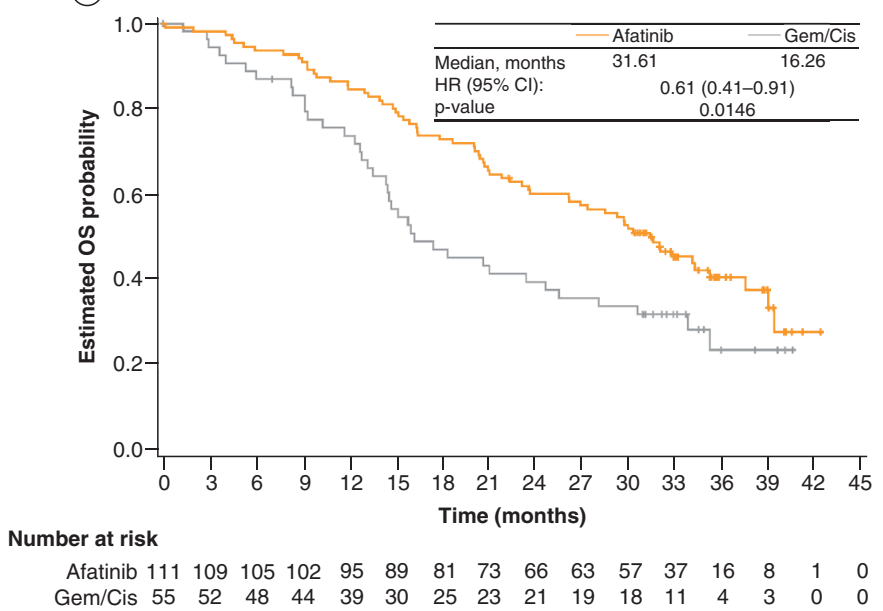

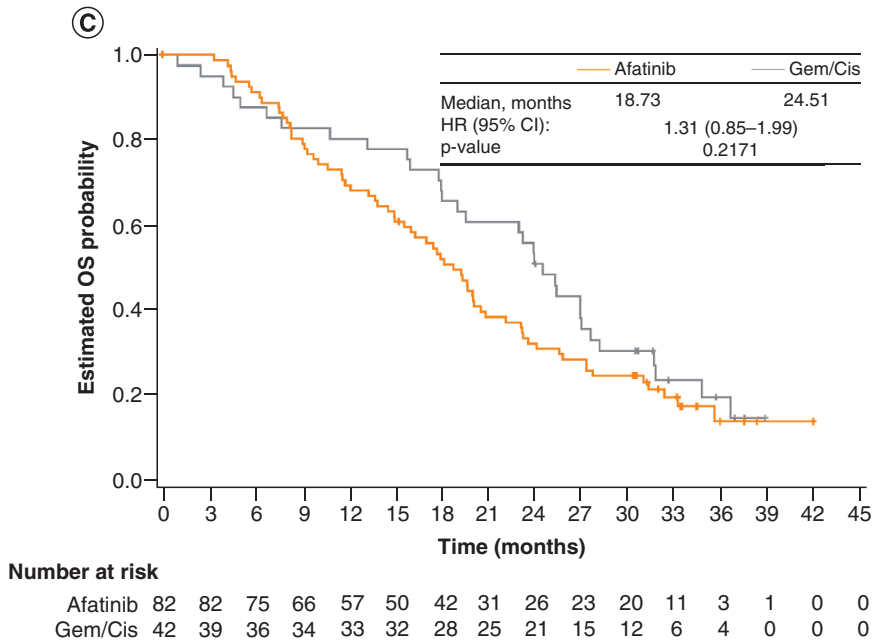

Figure 2. Overall survival in Chinese patients treated with afatinib compared with gemcitabine plus cisplatin in the LUX-Lung 6 study (independent review). (A) Overall population (median: 23.1 vs 23.2 months; $p=0.78$ ); (B) patients with Del19 mutations (median: 31.6 vs 16.3 months; $\mathrm{p}=0.015)$; $(\mathrm{C})$ patients with the L858R mutation (median: 18.7 vs $24.5 ; \mathrm{p}=0.22$ ).

Cis: Cisplatin; Gem: Gemcitabine; HR: Hazard ratio; OS: Overall survival.

Reproduced from [31], with permission from Dove Medical Press Ltd.

$\left[p_{\text {interaction }}=0.44\right] ;$ the HR was $0.95[0.67-1.33]$ and 0.78 [95\% CI: 0.52-1.17], respectively [30]. In the overall population, ORR, DCR and duration of response were all numerically higher with afatinib than gefitinib (Table 1); the difference in ORR between the arms was statistically significant $(p=0.0083)$ [25]. No comparisons for these end points have been made between Asian and non-Asian patients.

Results from a large, single-arm Phase IIIb study of afatinib in EGFR TKI-naive patients (study 1200.66; ClinicalTrials.gov: NCT01953913) provide further evidence of the efficacy of afatinib in Asian patients [33]. In total, 541 patients treated at 34 sites in China, Hong Kong, India, Singapore and Taiwan were included in the analysis; of these patients, more than three-quarters $(76.2 \%)$ were enrolled at sites in China. Afatinib demonstrated encouraging time to symptomatic progression (TTSP; median: 14.0 months [95\% CI: 12.9-15.9]) and PFS (median: 12.1 months [95\% CI: 11.0-13.6]) in patients with common and uncommon EGFR mutations. Median TTSP was longer than median PFS, which suggests that afatinib treatment may be continued beyond progression, reflecting real-world clinical practice and treatment guidelines [15,34,35].

Real-world data support the findings of prospective studies. While few studies in Chinese patients have been published, a recent analysis of 60 patients treated with afatinib (30\% with uncommon EGFR mutations and $40 \%$ with baseline brain metastases), showed median PFS of 12.3 months in patients receiving first-line afatinib [36]. A 
number of other real-world studies conducted in Taiwan also corroborate findings from clinical trials [37-40]; median PFS with afatinib was around 12 months in NSCLC patients with common EGFR mutations (L858R/Del19), and 11 to almost 20 months in patients with uncommon mutations [39,41].

\section{Long-term response to afatinib}

Data from the LUX-Lung 3, 6 and 7 studies indicate that afatinib is associated with long-term response of 3 years or longer in a proportion of patients [42]. In LUX-Lung 6, 10\% of afatinib-treated patients were long-term responders (treated with afatinib for $\geq 3$ years), with a median treatment duration of 56 months (range: 37-68 months). Baseline characteristics of the long-term responders were generally consistent with the overall patient populations, though there was a higher proportion of women and patients with Del19 mutations. Many long-term responders had tolerability-guided dose adjustments, with some patients successfully undergoing prolonged treatment at doses as low as $20 \mathrm{mg}$.

\section{Afatinib in patients with brain metastases}

Treatment with afatinib has been shown to confer clinical benefit in patients with brain metastases and may even slow central nervous system (CNS) progression compared with conventional chemotherapy. In LUX-Lung 6, median PFS in patients with baseline brain metastases was 8.2 months with afatinib compared with 4.7 months with gemcitabine and cisplatin (HR: 0.47 [95\% CI: 0.18-1.21]; $\mathrm{p}=0.11$ ) [43]. Furthermore, median time to CNS progression was longer with afatinib (15.2 months) than with chemotherapy ( 7.3 months). Patients in the 1200.66 study with brain metastases at baseline had slightly shorter median PFS (10.9 months) than those without baseline brain metastases (12.4 months), but still derived substantial benefit from afatinib [44].

In a Korean real-world study, 22 of 29 patients $(76 \%)$ with evaluable follow-up brain magnetic resonance imaging for nonirradiated brain metastatic lesions had a significant response to first-line afatinib [45]. Further, a Taiwanese retrospective analysis of 28 patients with lung adenocarcinoma, who were treated with first-line afatinib or whole brain radiotherapy followed by afatinib maintenance, reported ORR rates of 82 versus $88 \%$, respectively [46]. The complete response rate for intracranial lesions was notably higher in patients receiving afatinib monotherapy compared with whole brain radiotherapy plus afatinib maintenance (64 vs $18 \% ; \mathrm{p}=0.02$ ).

\section{Tolerability}

In the LUX-Lung trials, afatinib demonstrated a predictable safety profile, with adverse events (AEs) largely related to its mechanism of action, and generally consistent with the AEs reported for other EGFR TKIs (Table 1) $[22,23,25,31,47,48]$. Afatinib-related AEs were primarily gastrointestinal and dermatologic; the most frequently observed drug-related AEs in LUX-Lung 3, 6 and 7 were diarrhea, rash/acne and stomatitis/mucositis (Table 1). In the overall populations of LUX-Lung 3 and 6 , afatinib had a better tolerability profile than chemotherapy, with markedly lower rates of hematologic toxicity [22,23]. In the sub-analysis of Chinese patients in LUX-Lung 6, similar proportions of patients experienced grade $\geq 3$ treatment-related AEs in the afatinib and chemotherapy groups (36\% in each), but substantially more patients in the chemotherapy arm experienced grade 4 treatment-related AEs ( 21 vs $1 \%$ with afatinib) [31]. In LUX-Lung 7 , afatinib was associated with a higher incidence of grade $\geq 3$ treatment-related AEs, particularly diarrhea and rash, compared with gefitinib. However, elevated liver enzymes and interstitial lung disease were more common with gefitinib [25]. The safety profile of afatinib appeared to be similar between the overall population and the Chinese sub-population of LUX-Lung 6, with rash/acne, diarrhea and stomatitis/mucositis the most frequent treatment-related AEs (Table 1) [23,31]. Consistent with global studies of afatinib, diarrhea, rash/acne and stomatitis were also the most frequently reported grade $\geq 3$ afatinib-related AEs in the 1200.66 real-world study [33].

\section{Impact of tolerability-guided dose adjustment}

Afatinib is available in multiple dose strength formulations. Specific recommendations for tolerability-guided dose adjustment have been developed, and studies suggest that these do not negatively impact on efficacy in Asian populations [33,49]. In the 1200.66 study, around a quarter of patients had at least one dose reduction which effectively managed treatment-related AEs, including diarrhea and rash, such that treatment discontinuations were uncommon $(<10 \%)$. Neither PFS nor TTSP were affected by dose reduction during the first 6 months of treatment. PFS and TTSP in patients who did not undergo dose reduction were 11.3 and 14.7 months, respectively, compared with 14.1 and 17.7 months in patients who underwent dose reduction [49]. 
Similar PFS outcomes with or without dose reductions were also reported for LUX-Lung 6 [50], LUX-Lung 7 [51], a Taiwanese real-world analysis [40] and a global real-world study in which 44\% of patients were Asian [52]. In LUX-Lung 6, no significant difference in PFS was seen in patients who underwent afatinib dose reduction during the first 6 months of treatment compared with those who did not (median: 12.3 vs 11.0 months; HR 1.00 [95\% CI: 0.69-1.46]; $\mathrm{p}=0.982$; Figure 3A) [50]. Sixty-seven patients (28\%) treated with afatinib required dose reductions due to treatment-related AEs (Table 1), of whom 54 (81\%) experienced grade $\geq 3$ treatment-related AEs. After dose reduction, only $12 \%$ of patients had grade $\geq 3$ treatment-related AEs (Figure 3B) [50]. In LUX-Lung 7, there was no difference in the frequency of discontinuations due to treatment-related AEs between afatinib and gefitinib (6\% in each arm; Table 1), but there were more dose reductions due to AEs in patients receiving afatinib (42 vs $2 \%$ ), likely due to the well-defined dose adjustment protocol for afatinib and the fact that gefitinib is only available in one dose formulation [25]. As with LUX-Lung 6, there was no significant difference in PFS in patients who did or did not undergo dose reduction within the first 6 months (median: 12.8 vs 11.0 months; HR 1.34 [95\% CI: $0.90-2.00$ ]; $\mathrm{p}=0.144$ ) [51]. In the Taiwanese real-world study, patients receiving afatinib doses of $40 \mathrm{mg}$ during the first 6 months of treatment had a median PFS of 12.0 months, compared with 11.0 months in patients who received <40 mg (HR: 0.84 [95\% CI: 0.53-1.31]; p > 0.05) [40].

The effectiveness of tolerability-guided dose reduction of afatinib for the management of AEs reflect its pharmacokinetic characteristics. Afatinib shows moderate-to-high interpatient variability in exposure [53]. Key covariates of increased exposure include lower bodyweight, female sex and age [53]. AEs tend to be more common in patients with higher initial plasma concentrations [50]. Dose reduction reduces excessive plasma concentrations, thereby mitigating treatment-related AEs [50]. While dose reduction does not appear to impact efficacy, it is important not to adapt starting dose based on clinical characteristics because there are no prospective data to support a $<40 \mathrm{mg}$ starting dose and under-dosing may be a possibility.

\section{Patient-reported outcomes}

Assessment of patient-reported outcomes (PROs) is an important complement to efficacy and tolerability data and can help provide a broader picture of the impact of treatment at an individual patient level. For example, PRO data can provide evidence on whether AEs associated with targeted therapy impact quality of life. In LUX-Lung 6, global health status/quality of life (measured using the European Organisation for Research and Treatment of Cancer [EORTC] Quality of Life Core Questionnaire C30 [QLQ-C30]) was improved with afatinib compared with chemotherapy, including in an exploratory analysis of patients with common EGFR mutations (Del19 and/or L858R) [31,54,55]. In Chinese patients treated in LUX-Lung 6, significantly greater proportions of patients in the afatinib group had improvements in cough, dyspnea and pain than in the gemcitabine/cisplatin group; time to deterioration of cough, dyspnea and pain were also delayed with afatinib [31].

Although better PFS and OS outcomes with afatinib relative to chemotherapy have been seen in patients with Del19 mutations than in those with L858R [23,31], PRO data from the overall LUX-Lung 6 population suggest that, for cough and dyspnea, afatinib confers similar benefit in patients with Del19 and L858R [55]. Similar improvements in health-related quality of life, as assessed by the EuroQol 5 dimensions questionnaire (EQ-5D) and the EuroQol Visual Analog Scale (EQ-VAS), were observed with afatinib and gefitinib in LUX-Lung 7; notably, quality of life was not affected by dose reductions during the first 6 months of treatment in patients treated with afatinib $[51,56]$.

\section{Mechanisms of acquired resistance to afatinib \& subsequent therapy}

Despite the impressive initial efficacy of afatinib and other EGFR TKIs in EGFRm + NSCLC, resistance to treatment eventually develops in most patients. In general, resistance mechanisms to first-generation EGFR TKIs have been more comprehensively studied than resistance mechanisms to afatinib. The predominant resistance mechanism to the first-generation EGFR TKIs is the T790M mutation in EGFR [57], which is detected in approximately 50-70\% of patients overall and is more prevalent in patients with Del19-positive tumors than L858R-positive tumors [58-60]. A number of studies indicate that the T790M emergence rate is similar in patients treated with afatinib as it is with patients treated with gefitinib/erlotinib, ranging from 40 to 73\% [37,58,61-64]. Few data, however, are available regarding the T790M acquisition rate in Chinese NSCLC patients. Additional mechanisms of resistance to EGFR TKIs have largely been identified based on studies of erlotinib and gefitinib and include MET amplification (5-20\%), transformation to small cell lung cancer ( $\sim 10 \%)$, epithelial mesenchymal transition (EMT; 1-2\%), mutations in PI3K (2-3\%), BRAF $(\sim 1 \%)$ or KRAS ( 1\%) and other secondary mutations in EGFR $(\sim 1 \%)[65,66]$. Up to $10-15 \%$ of patients who become resistant to first-generation EGFR TKIs demonstrated amplification of 
(A)

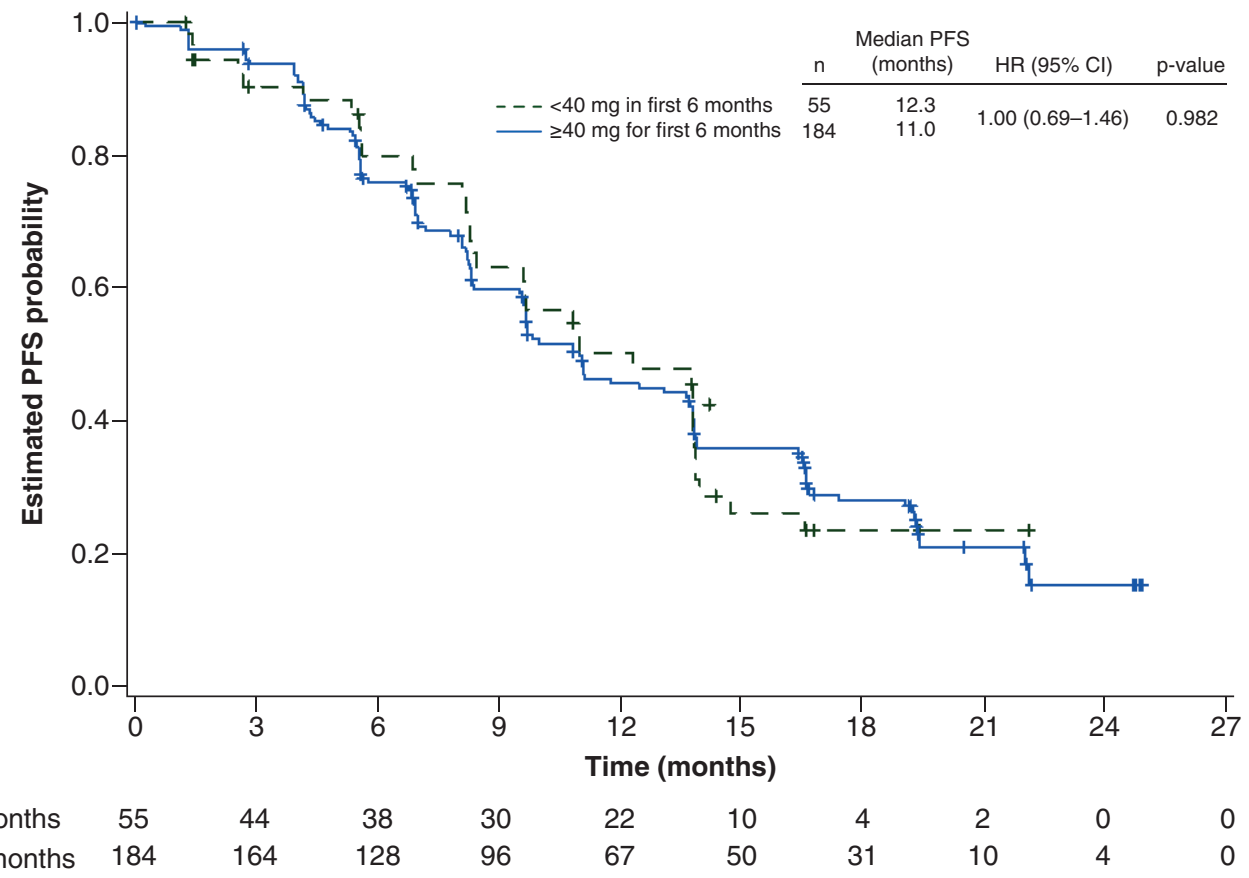

(B)

Number at risk $<40 \mathrm{mg}$ in first 6 months $\geq 40 \mathrm{mg}$ for first 6 months

$\begin{array}{cccc}55 & 44 & 38 & 30 \\ 184 & 164 & 128 & 96\end{array}$

22
67

50

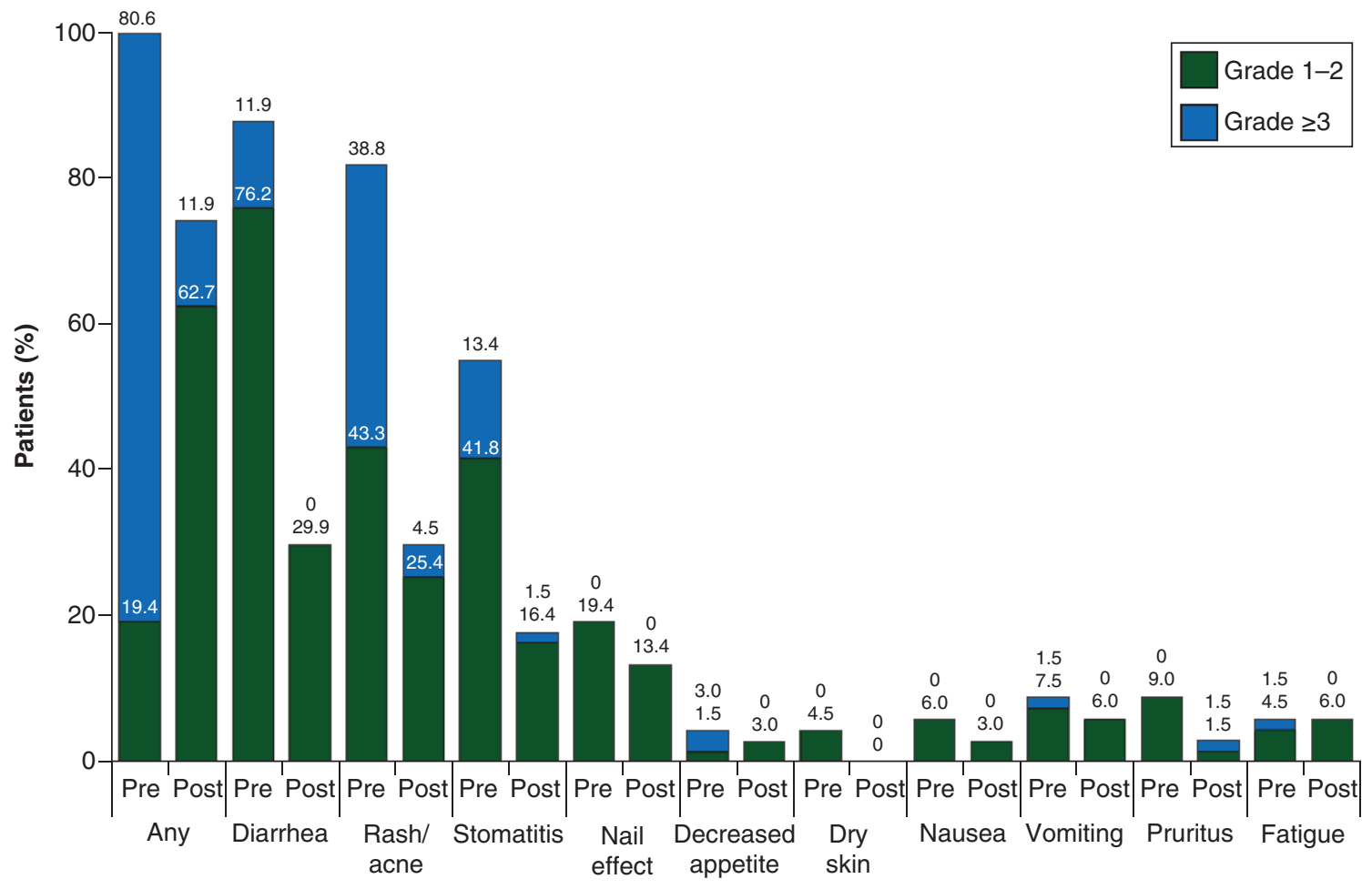

Figure 3. Impact of dose reduction on progression-free survival and adverse events in patients receiving afatinib in the LUX-Lung 6 study. (A) PFS in patients who had dose reductions within the first 6 months, and those who remained on afatinib $\geq 40 \mathrm{mg}$ once daily; (B) most common treatment-related adverse events pre- and post-afatinib dose reduction.

HR: Hazard ratio; PFS: Progression-free survival.

Reproduced from [50], ( ) 2016, with permission from Elsevier. 
HER2 [65,66]. However, it is unlikely HER2 amplification is a major mechanism of resistance to afatinib given that it is an ErbB family blocker. Emerging evidence indicates that many (up to 46\%) of EGFR TKI-resistant tumors demonstrate co-occuring mechanisms of resistance based on next-generation sequencing of blood-derived cell-free tumor DNA [66-69]. Again, these studies have predominantly been based on studies of erlotinib or gefitinib. Further data are required to elucidate mechanisms of resistance to afatinib.

In patients with T790M-positive disease following progression on a first-line EGFR TKI, treatment with second-line osimertinib, which is approved in this setting, produces high response rates and prolonged PFS (median: $\sim 10-12$ months) [58,70]. Data from retrospective analyses suggest that the use of osimertinib in patients with T790M-positive disease progressing after first-line afatinib can result in promising clinical outcomes in patients with EGFRm + NSCLC [61,71]. In a global, observational, study (GioTag) of 204 patients treated with sequential afatinib and osimertinib, overall time on treatment was over 2 years (median: 27.6 months) [71]. Time on treatment was particularly striking in Asian patients (nearly 4 years) and patients with tumors harboring EGFR Del19 mutations. A recent updated analysis of GioTag demonstrated median OS of 41.3 months overall (90\% CI: 36.8-46.3) and 45.7 months (90\% CI: 45.3-51.5) in those with Del19-positive tumors [72]. OS in Asian patients was immature. In a post-hoc analysis of data from 37 patients in LUX-Lung 3, 6 and 7 who discontinued afatinib and received subsequent osimertinib, of whom $54 \%$ were Asian, median time on osimertinib in any treatment line was 20.2 months (95\% CI: 12.8-31.5), and median OS had not been reached at the time of analysis [73]. In ten patients who received consecutive afatinib and osimertinib, median PFS-2 (the time from initiation of afatinib to the last day of osimertinib therapy) was 53.3 months. Together, these findings illustrate that sequential use of EGFR TKIs can lead to prolonged periods of chemotherapy-free treatment in patients with EGFRm+ NSCLC. Moreover, recent findings suggest that outcomes may be particularly favorable if second- and third-generation TKIs are used sequentially. In a Japanese retrospective analysis, ORR with osimertinib was higher in patients with $E G F R \mathrm{~m}+$ NSCLC who had previously received afatinib than those who had previously received a first-generation EGFR TKI (83 vs 54\%; $\mathrm{p}=0.007$ ), as was DCR (91 vs $71 \%$; $\mathrm{p}=0.03$ ) [74]. Further, median PFS with osimertinib was numerically longer in afatinib-treated patients than in those treated with a first-generation EGFR TKI (15.6 vs 8.9 months; $\mathrm{p}=0.20$ ).

A possible shortcoming of sequential EGFR TKI therapy is that it is only applicable to patients who develop T790M-dependent acquired resistance to first-line therapy. Currently, subsequent therapies for patients who receive first-line afatinib and progress due to a T790M-independent mechanism are limited, with platinumdoublet chemotherapy being the principal treatment option in these patients [14]. However, in LUX-Lung 3, 6 and 7 studies, there was a high uptake of subsequent therapies $(71 \% ; 64 \%$ in Chinese patients) following progression on or after first-line afatinib, indicating that clinicians and patients are motivated to continue treating EGFR TKI-resistant disease even without targeted therapy [73]. Moreover, recent data suggest that targeting alternative signaling pathways, either as monotherapy or in combination with chemotherapy, an EGFR TKI or an immune checkpoint inhibitor may be applicable for some patients, including those with T790M-independent resistance to first-line EGFR TKIs [75,76]. Potential treatment options in development for T790M-negative tumors have recently been reviewed in detail elsewhere [76]. In brief, a number of targeted agents could be considered for patients with T790M-negative tumors following failure of afatinib, depending on whether a specific resistance mechanism is identified. For example, in the case of MET amplification a clinical trial with a selective MET inhibitor, such as capmatinib, tepotinib or savolitinib could be considered [76]. Alternatively, simultaneous targeting of EGFR and MET with a bi-specific antibody is another potential approach for the treatment of disease that has relapsed following prior EGFR TKI therapy [77]. In patients with AXL activation (associated with EMT) several AXL blockers are in clinical development, including DS-1205c, bemcentinib (BGB324) and dubermatinib (TP-0903) [76]. Also in the case of BRAF or PI3K mutations, clinical trials with MEK inhibitors (e.g., selumetinib) or mTOR inhibitors (e.g., sapanisertib), respectively, may be appropriate [76].

Immune checkpoint inhibitors could play a role post afatinib. For example, in a sub-analysis of the triple-arm, Phase III IMpower study in patients with EGFRm + disease, addition of the anti-programmed death ligand-1 (PD-L1) antibody, atezolizumab, to bevacizumab plus chemotherapy significantly improved OS (median: not evaluable vs 17.5 months; HR: 0.31 [95\% CI: 0.11-0.83]), and PFS (median: 10.3 vs 6.1 months; HR: 0.41 [95\% CI: 0.23-0.75]) versus bevacizumab plus chemotherapy alone [75]. The combination was associated with a treatment-related grade $\geq 3 \mathrm{AE}$ rate of $57 \%$. However, the combination of an EGFR TKI with an immune checkpoint PD-(L)1 blocker could potentially lead to severe immune-related AEs. For example, the Phase IB TATTON trial (second-line osimertinib plus durvalumab) was terminated due to a high incidence of interstitial 
lung disease [78]. On the other hand, while few data are currently available in NSCLC, a Phase II study of afatinib and pembrolizumab in patients with head and neck cancer indicated that this combination may be feasible, with no recorded cases of pneumonitis [79]. Sequential regimens of an EGFR TKI followed by a PD-(L)1 blocker do not appear to be associated with immune-related AEs in patients with EGFR mutation-positive NSCLC [80].

In summary, in patients with $E G F R$ mutation-positive NSCLC treated with first-line afatinib, second-line osimertinib appears to be a promising approach in patients with T790M-mediated resistance. With respect to T790M-independent mechanisms of resistance to afatinib, and potential second-line treatment approaches, more data are required.

\section{Afatinib compared with other EGFR TKIs in Chinese patients}

Current CSCO clinical guidelines do not provide specific recommendations regarding the preferred first-line EGFR TKI, leaving the decision to individual clinicians. Many factors may be considered when choosing a firstline treatment, including the type of EGFR mutation, whether brain metastases are present, the patient's preference, age and performance status, the efficacy and safety profile of each agent and its impact on PROs, subsequent treatment options and the cost of therapy. Table 2 summarizes prospective clinical trial data assessing the efficacy and safety of EGFR TKIs in Chinese patients (or in broader Asian populations that include Chinese patients, if China-specific data were not available). While it is not possible to directly compare independent clinical trials, these studies may provide some insight into the relative efficacy and safety profiles of the EGFR TKIs in Chinese patients.

Median PFS was largely similar in each study, ranging from 9.8 to 17.8 months (Table 2). The longest PFS was observed with osimertinib. Sub-analyses of the FLAURA trial in Asian patients (14\% of whom were Chinese) demonstrated a significant improvement in PFS versus gefitinib or erlotinib (HR: 0.54 [95\% CI: 0.41-0.72] [82]), although the magnitude of the effect was less than observed in non-Asian patients (HR: 0.34) [26]. In a further sub-analysis, significant PFS benefit was also observed in Chinese patients (median: 17.8 vs 9.8 months; HR: 0.56 [95\% CI: $0.37-0.85]$ ] [83]. In the only head-to head trial undertaken exclusively in Chinese patients, CTONG 0901, there was no difference in PFS between erlotinib and gefitinib (HR: 0.81 [95\% CI: 0.62-1.05] [81]).

Median OS across studies ranged from 20.1 to 37.1 months. Again, median OS was highest with osimertinib. However, interestingly, unlike the overall FLAURA dataset [26], there was no evidence of an OS benefit with osimertinib versus gefitinib/erlotinib in Asian patients (HR: 1.00 [95\% CI: 0.75-1.32]) [29]. There was also no evidence of OS benefit between afatinib and gefitinib in Asian patients (HR: 0.95 [95\% CI: 0.67-1.33]) [30] or between erlotinib and gefitinib in Chinese patients (HR: 0.84 [95\% CI: 0.63-1.13]) [81]. Based on OS, therefore, it is unclear which first-line EGFR TKI may be most effective in Chinese, or Asian, patients. Of note, as osimertinib has only recently become available, few patients in any of the listed trials would have received second-line osimertinib following failure of another EGFR TKI. Given encouraging clinical activity observed in GioTag, and other studies (discussed previously), it is possible that sequential EGFR TKI regimens could confer prolonged OS (in patients with T790M-mediated acquired resistance) compared with what has been observed to date. This possibility requires prospective evaluation in clinical trials.

When considering the optimal use of EGFR TKIs in patients with EGFRm + NSCLC, in China and elsewhere, it is also important to assess recent progress regarding EGFR-TKI-based combination strategies. Several novel combinations, such as erlotinib plus ramucirumab [84], gefitinib plus pemetrexed-carboplatin [85,86] and erlotinib plus bevacizumab [87], have demonstrated promising outcomes with significant PFS benefit and, in some cases [85,86], OS benefit observed in randomized trials. In one trial, the latter combination conferred OS of over 50 months [86]. These findings indicate that novel combinations of existing drugs may be able to achieve similar or better outcomes than single targeted agents in EGFRm + NSCLC.

It is important to consider tolerability profiles when deciding upon first-line treatment in individual patients. As discussed, while EGFR TKIs have overlapping, class-based tolerability profiles, available data indicate that there are qualitative and quantitative differences in the frequency of grade AEs with different TKIs. Although afatinib was associated with a relatively high incidence of grade $\geq 3$ rash/acne, diarrhea and stomatitis in clinical trials and realworld studies, certain other grade $\geq 3$ AEs may be more prevalent with first-generation EGFR TKIs (e.g., elevated AST/ALT). Furthermore, while osimertinib has a generally favorable tolerability profile, as it spares wild-type EGFR, certain grade $\geq 3$ AEs occurred that appeared to be less prevalent with other EGFR TKIs (e.g., prolonged QT interval). 


\begin{tabular}{|c|c|c|c|c|c|c|c|}
\hline EGFR TKI & Trial & $n$ & Median PFS (months) & Median OS (months) & ORR (\%) & Most common grade $\geq 3$ AEs $(\%)^{\dagger}$ & Ref. \\
\hline \multirow[t]{2}{*}{ Afatinib $\ddagger$} & LUX-Lung 6 & $193^{\S}$ & $11.0^{\S}$ & $23.6^{\S}$ & $69^{\S}$ & $\begin{array}{l}\text { Any: } 36 \\
\text { Rash/acne: } 16 \\
\text { Stomatitis: } 6 \\
\text { Diarrhea: } 6\end{array}$ & [31] \\
\hline & LUX-Lung $7 \mathbb{I}$ & $94^{\#}$ & $11.0^{\dagger \dagger}$ & $27.9^{\dagger \dagger}$ & NR & $\begin{array}{l}\text { Any: } 31 \\
\text { Diarrhea: } 13 \\
\text { Rash/acne: } 9 \\
\text { Fatigue: } 6\end{array}$ & {$[25,30]$} \\
\hline \multirow[t]{3}{*}{ Erlotinib } & ENSURE & 110 & 11.0 & 26.3 & 63 & $\begin{array}{l}\text { Any: } 44 \\
\text { Rash: } 6\end{array}$ & [18] \\
\hline & OPTIMAL & 83 & 13.1 & 22.8 & 82 & $\begin{array}{l}\text { Any: } 17 \\
\text { Increased ALT: } 4 \\
\text { Rash: } 2\end{array}$ & [11] \\
\hline & CTONG 0901 & 128 & 13.0 & 22.9 & 56 & $\begin{array}{l}\text { Any: } 5 \\
\text { Rash: } 2 \\
\text { Increased bilirubin: } 2\end{array}$ & [81] \\
\hline \multirow[t]{2}{*}{ Gefitinib } & CTONG 0901 & 128 & 10.4 & 20.1 & 52 & Any: 2 & [81] \\
\hline & LUX-Lung $7 \mathbb{I}$ & $88^{\#}$ & 11.0 & 24.5 & NR & $\begin{array}{l}\text { Any: } 18 \\
\text { Increased ALT/AST: } 8 \\
\text { Rash/acne: } 3 \\
\text { ILD: } 2\end{array}$ & {$[25,30]$} \\
\hline \multirow[t]{2}{*}{$\begin{array}{l}\text { Erlotinib or } \\
\text { gefitinib }\end{array}$} & FLAURA $\$ \S, \#$ & $160^{\#}$ & 11.0 & 35.8 & 75 & $\begin{array}{l}\text { Any: } 48 \\
\text { Increased AST: } 6 \\
\text { Diarrhea: } 3 \\
\text { Decreased appetite: } 3\end{array}$ & {$[29,82]$} \\
\hline & $\begin{array}{l}\text { FLAURA (Chinese } \\
\text { only)I I }\end{array}$ & 65 & 9.8 & NR & 75 & $\begin{array}{l}\text { Any: } 23 \\
\text { Increased ALT: } 6\end{array}$ & [83] \\
\hline \multirow[t]{2}{*}{ Osimertinib } & FLAURA $\$ \$, \#$ & $162^{\#}$ & 16.5 & 37.1 & 80 & $\begin{array}{l}\text { Any: } 40 \\
\text { Decreased appetite: } 3 \\
\text { Prolonged QT: } 3\end{array}$ & {$[29,82]$} \\
\hline & $\begin{array}{l}\text { FLAURA (Chinese } \\
\text { only) } \mathbb{\Psi}\end{array}$ & 71 & 17.8 & NR & 83 & $\begin{array}{l}\text { Any: } 49 \\
\text { Decreased neutrophil count: } 6 \\
\text { Decreased WBC/lymphocyte count: } \\
\text { 4/4 } \\
\text { Prolonged QT: } 4 \\
\text { Elevated AST: } 4\end{array}$ & [83] \\
\hline Icotinib & CONVINCE & 148 & 11.2 & 30.5 & NR & $\begin{array}{l}\text { Rash: } 15 \\
\text { Diarrhea: } 7\end{array}$ & [24] \\
\hline
\end{tabular}

$\dagger$ Top three AEs listed (if over $1 \%$ frequency).

¥Treatment-related AEs reported in LUX-Lung 6 and 7; treatment-emergent AEs reported for other trials.

\&patients with common EGFR mutations.

IThere was a trend toward improved PFS with afatinib vs gefitinib in Asian patients in LUX-Lung 7 (HR: 0.76; $95 \%$ CI: 0.54-1.06); there was no significant difference in OS (HR: 0.94; 95\% Cl: 0.67-1.33).

\#Asian patients.

†† Data on file.

¥No significant difference in PFS (HR: $0.81 ; 95 \% \mathrm{Cl}: 0.62-1.05)$ or OS (HR: $0.84 ; 95 \% \mathrm{Cl}: 0.63-1.13)$ between erlotinib and gefitinib in CTONG 0901.

$\S \S$ There was a significant improvement in PFS with osimertinib vs gefitinib/erlotinib in Asian patients in FLAURA (HR: 0.54; $95 \%$ CI: $0.41-0.72)$; there was no significant difference in OS (HR: $1.00 ; 95 \% \mathrm{Cl}: 0.75-1.32)$.

IIThere was a significant improvement in PFS with osimertinib vs gefitinib/erlotinib in Chinese patients in FLAURA (HR: 0.56; 95\% Cl: 0.37-0.85).

AE: Adverse event; HR: Hazard ratio; ILD: Interstitial lung disease; NR: Not reached; NSCLC: Non-small-cell lung cancer; ORR: Overall response rate; OS: Overall survival; PFS: Progression-free survival; TKI: Tyrosine kinase inhibitor; WBC: White blood cell.

\section{Conclusion \& future perspective}

Afatinib is an effective first-line treatment option in Chinese patients with $E G F R \mathrm{~m}+\mathrm{NSCLC}$. Its safety profile is predictable, with drug-related AEs being manageable with supportive care and tolerability-guided dose adjustments. Such dose adjustments do not appear to compromise the efficacy of afatinib, but rather, may help to facilitate longterm continuation of active treatment. While second- and third-generation EGFR TKIs are both superior to first-generation TKIs, no prospective data are available comparing second- and third-generation EGFR TKIs in China or, indeed, in any population. Therefore, it remains unclear what the optimal treatment should be. On one hand, osimertinib is an attractive first-line treatment option in Chinese patients based on the FLAURA data. However, resistance to osimertinib is mediated by a diverse range of mechanisms [88], and currently no targeted 
agents are available that specifically address these resistance mechanisms. Consequently, an alternative sequencing strategy, where osimertinib is reserved for second-line use following failure of afatinib, warrants consideration in Chinese patients with $E G F R \mathrm{~m}+\mathrm{NSCLC}$ as $50-70 \%$ of these patients will develop T790M-mediated resistance. Data from GioTag, showing a combined time on treatment of nearly 4 years in Asian patients [71], highlight the potential of this strategy, which warrants further investigation in patients with T790M-positive tumors. Also, combination strategies are of potential interest for example second- or third-generation EGFR TKIs combined with anti-angiogenic agents or low toxicity chemotherapy. Furthermore, more data are required to assess how EGFR mutation subtype, and concurrent genetic alterations, influence the response to different treatment regimens.

Increased availability of effective treatments for EGFR mutation-positive NSCLC highlight the importance of rebiopsy (tissue and/or blood) and NGS at the point of tumor progression to inform subsequent treatment decisions. Treatment options for patients with T790M-independent mechanisms of resistance remain an unmet medical need. However, ongoing clinical development programs with agents including MET, MEK and mTOR inhibitors, and novel combination strategies including immune checkpoint inhibitors, will hopefully identify individualized therapeutic strategies depending on the precise molecular signature of tumors following acquired resistance to afatinib.

In the absence of strong comparative data, further study is required to determine the most appropriate sequencing of EGFR TKIs that will maximize clinical benefit. The ongoing European APPLE study comparing first-line osimertinib with first-line gefitinib followed by osimertinib at progression may provide some insight into this issue [89], but similar studies should be undertaken in Asia. In addition, further research into patient-specific factors that may influence response to specific targeted therapies is needed. Additional investigation is also needed to establish suitable treatment options for patients who progress on first-line osimertinib treatment.

As the majority of patients with EGFRm + NSCLC go on to receive second or later lines of therapy, maximizing the clinical benefits associated with each treatment line is essential, so that patients are able to live extended lives while maintaining quality of life.

\section{Executive summary}

\section{Background}

- Mutations in EGFR are highly prevalent in Chinese patients with NSCLC, being detected in 35-50\% of individuals.

- Currently, the treatment of EGFR mutation-positive (EGFRm+) NSCLC in China generally follows the guidelines issued by the Chinese Society of Clinical Oncology (CSCO).

- Six EGFR tyrosine kinase inhibitor (TKIs) are currently approved in China for the first-line treatment of EGFRm+ NSCLC: erlotinib; gefitinib; icotinib; afatinib; dacomitinib and osimertinib.

- This article summarizes available clinical trial and real-world data on the efficacy and tolerability of afatinib, and the impact of dose adjustments, in Chinese patients. In addition, we provide a general comparison of the efficacy and safety of the EGFR TKIs in Chinese patients.

Afatinib in Chinese patients: clinical data

Efficacy

- In the Phase III LUX-Lung 6 trial (almost 90\% Chinese patients), afatinib significantly improved progression-free survival (PFS) versus platinum-based chemotherapy. Afatinib significantly prolonged OS versus chemotherapy in patients with Del19-positive tumors. Similar findings were observed in a sub-analysis of Chinese patients.

Approximately $10 \%$ of afatinib-treated patients were long-term responders (treated with afatinib for $\geq 3$ years).

- In the Phase Ilb LUX-Lung 7 trial (>50\% Asian patients; $15 \%$ Chinese patients), afatinib significantly improved PFS and time-to-treatment failure versus gefitinib, with no significant differences between Asian and non-Asian patients.

- In a large, single-arm Phase IIIb study of afatinib in EGFR TKI-naive patients (1200.66; $>75 \%$ Chinese patients), afatinib conferred encouraging time to symptomatic progression and PFS in patients with both common and uncommon EGFR mutations.

- Few real-world studies of afatinib in Chinese patients have been published, but available data corroborate findings from clinical trials.

- Sub-analyses of LUX-Lung 6 and 1200.66 indicate that afatinib confers clinical benefit in Chinese patients with brain metastases and may slow CNS progression compared with conventional chemotherapy.

Tolerability

- Consistent with global studies, the most frequent treatment-related adverse events (AEs) in Chinese patients treated with afatinib in LUX-Lung 6 and 1200.66 were diarrhea, rash/acne and stomatitis.

- Based on available data, tolerability-guided dose reductions reduced the frequency and severity of AEs without impacting on efficacy in Chinese patients. 
- In Chinese patients treated in LUX-Lung 6, significantly greater proportions of patients in the afatinib group had improvements in cough, dyspnea and pain than in the chemotherapy group; time to deterioration of cough, dyspnea and pain were also delayed with afatinib.

Mechanisms of acquired resistance to afatinib \& subsequent therapy

- The predominant resistance mechanism to the first- and second-generation EGFR TKIs is the T790M mutation in $E G F R$, which is detected in approximately $50-70 \%$ of patients.

- Data from retrospective analyses suggest that the use of osimertinib in patients with T790M-positive disease progressing after first-line afatinib can result in promising clinical outcomes in patients with EGFRm+ NSCLC.

- In a global, observational, study (GioTag) of 204 patients treated with sequential afatinib and osimertinib, median time-to-treatment failure was over 2 years (near 4 years in Asian patients) and median OS was 41.3 months.

- Currently, subsequent therapies for patients who receive first-line afatinib and progress due to a T790M-independent mechanism are limited, with platinum-doublet chemotherapy being the principal treatment option in these patients.

Afatinib compared with other EGFR TKIs in Chinese patients

- Current CSCO guidelines do not provide specific recommendations regarding the preferred first-line EGFR TKI, leaving the decision to individual clinicians.

- In prospective clinical trials, median PFS achieved with different EGFR TKIs in Chinese patients ranged from 9.8 to 17.8 months.

- The longest PFS was observed with osimertinib in the Phase III FLAURA trial, although the magnitude of benefit over gefitinib/erlotinib appeared to be greater in non-Asian than Asian patients.

- Median OS across studies ranged from 20.1 to 37.1 months. No EGFR TKI has shown a significant OS benefit over another EGFR TKI in Asian or Chinese patients.

- Little prospective data are available assessing clinical outcomes in patients received second-line osimertinib following failure of another EGFR TKI.

- Novel combinations of existing drugs (e.g., an EGFR TKI plus chemotherapy) may be able to achieve similar or better outcomes than single targeted agents in EGFRm+ NSCLC.

- Although EGFR TKIs have overlapping, class-based tolerability profiles, available data indicate that there are qualitative and quantitative differences in the frequency of grade AEs with different, for example, afatinib was associated with a relatively high incidence of grade $\geq 3$ rash/acne, diarrhea and stomatitis.

- Osimertinib has a generally favorable tolerability profile, as it spares wild-type EGFR. Nevertheless, certain grade $\geq 3$ AEs appeared to be less prevalent with other EGFR TKIs (e.g., prolonged QT interval).

Conclusion \& future perspective

- Afatinib is an effective and tolerable first-line treatment option in Chinese patients with EGFRm+ NSCLC.

- No prospective data are available comparing second- and third-generation EGFR TKIs in China or, indeed, in any population.

- Osimertinib is an attractive first-line treatment option in Chinese patients based on the FLAURA data. However, as treatment options are limited following failure of osimertinib, it may be better to withhold it for second-line use in patients will develop T790M-mediated resistance.

- In the absence of strong comparative data, further study is required to determine the most appropriate sequencing of EGFR TKIs that will maximize clinical benefit in China.

\section{Author contributions}

The authors were fully responsible for all content and editorial decisions, were involved at all stages of manuscript development and have approved the final version.

\section{Acknowledgments}

We thank the patients, their families and all of the investigators who participated in the studies discussed in this paper.

Financial \& competing interests disclosure

Y-L Wu reports grants from AstraZeneca, Roche, Boehringer Ingelheim and personal fees from AstraZeneca, Roche, Boehringer Ingelheim, Pfizer, Eli Lilly, BMS, MSD and Sanofi. H-Y Tu reports no conflicts of interest. The authors have no other relevant affiliations or financial involvement with any organization or entity with a financial interest in or financial conflict with the subject matter or materials discussed in the manuscript apart from those disclosed.

Medical writing assistance, supported financially by Boehringer Ingelheim, was provided by Natalie Grainger and Caroline Perry of GeoMed, an Ashfield company, part of UDG Healthcare plc, during the preparation of this manuscript. 
Open access

This work is licensed under the Attribution-NonCommercial-NoDerivatives 4.0 Unported License. To view a copy of this license, visit http://creativecommons.org/licenses/by-nc-nd/4.0/

\section{References}

Papers of special note have been highlighted as: $\bullet$ of interest; $\bullet \bullet$ of considerable interest

1. The Global Cancer Observatory. GLOBOCAN 2018 population fact sheet: China (2018).

https:/gco.iarc.fr/today/data/factsheets/populations/160-china-fact-sheets.pdf2018

2. Zhou C. Lung cancer molecular epidemiology in China: recent trends. Transl. Lung Cancer Res. 3, 270-279 (2014).

3. Lindeman NI, Cagle PT, Beasley MB et al. Molecular testing guideline for selection of lung cancer patients for EGFR and ALK tyrosine kinase inhibitors: guideline from the College of American Pathologists, International Association for the Study of Lung Cancer, and Association for Molecular Pathology. J. Mol. Diagn. 15, 415-453 (2013).

4. Zhou Y, Yang Y, Yang C et al. Epidermal growth factor receptor (EGFR) mutations in non-small cell lung cancer (NSCLC) of Yunnan in southwestern China. Oncotarget 8, 15023-15033 (2017).

5. Cheng Y, Wang Y, Zhao J et al. Real-world EGFR testing in patients with stage IIIB/IV non-small-cell lung cancer in north China: a multicenter, non-interventional study. Thorac. Cancer 9, 1461-1469 (2018).

6. Lin D. Mutation profile of EGFR gene in Chinese patients with non-small-cell lung cancer (NSCLC): an analysis of 2,666 cases. J. Thorac. Oncol. 12, S1881 (2017).

7. Zhou J, Song XB, He H et al. Prevalence and clinical profile of EGFR mutation in non-small-cell lung carcinoma patients in southwest China. Asian Pac. J. Cancer Prev. 17, 965-971 (2016).

8. Wei WE, Mao NQ, Ning SF et al. An analysis of EGFR mutations among 1506 cases of non-small cell lung cancer patients in Guangxi, China. PLoS ONE 11, e0168795 (2016).

9. Quan X, Gao H, Wang Z et al. Epidermal growth factor receptor somatic mutation analysis in 354 Chinese patients with non-small cell lung cancer. Oncol. Lett. 15, 2131-2138 (2018).

10. Zhang YL, Yuan JQ, Wang KF et al. The prevalence of EGFR mutation in patients with non-small cell lung cancer: a systematic review and meta-analysis. Oncotarget 7, 78985-78993 (2016).

11. Zhou C, Wu YL, Chen G et al. Erlotinib versus chemotherapy as first-line treatment for patients with advanced EGFR mutation-positive non-small-cell lung cancer (OPTIMAL, CTONG-0802): a multicentre, open-label, randomised, phase 3 study. Lancet Oncol. 12, 735-742 (2011).

12. Shi Y, Li J, Zhang S et al. Molecular epidemiology of EGFR mutations in Asian patients with advanced non-small-cell lung cancer of adenocarcinoma histology - mainland China subset analysis of the PIONEER study. PLoS ONE 10, e0143515 (2015).

13. Tu HY, Ke EE, Yang JJ et al. A comprehensive review of uncommon EGFR mutations in patients with non-small cell lung cancer. Lung Cancer 114, 96-102 (2017)

14. Chinese Society of Clinical Oncology. Guidelines for advanced lung cancer therapy 2017 (2018). https://memoinoncology.com/congress-reports/csco/optimal-strategy-treatment-egfr-mutant-lung-cancer/

15. Wu YL, Planchard D, Lu S et al. Pan-Asian adapted Clinical Practice Guidelines for the management of patients with metastatic non-small-cell lung cancer: a CSCO-ESMO initiative endorsed by JSMO, KSMO, MOS, SSO and TOS. Ann. Oncol. 30, 171-210 (2019).

16. Shi Y, Sun Y, Ding C et al. China experts consensus on icotinib for non-small cell lung cancer treatment (2015 version). J. Thorac. Dis. 7, E468-472 (2015).

17. Rosell R, Carcereny E, Gervais R et al. Erlotinib versus standard chemotherapy as first-line treatment for European patients with advanced EGFR mutation-positive non-small-cell lung cancer (EURTAC): a multicentre, open-label, randomised phase 3 trial. Lancet Oncol. 13, 239-246 (2012).

18. Wu YL, Zhou C, Liam CK et al. First-line erlotinib versus gemcitabine/cisplatin in patients with advanced EGFR mutation-positive non-small-cell lung cancer: analyses from the phase III, randomized, open-label, ENSURE study. Ann. Oncol. 26, 1883-1889 (2015).

19. Mok TS, Wu YL, Thongprasert S et al. Gefitinib or carboplatin-paclitaxel in pulmonary adenocarcinoma. N. Engl. J. Med. 361, 947-957 (2009).

20. Maemondo M, Inoue A, Kobayashi K et al. Gefitinib or chemotherapy for non-small-cell lung cancer with mutated EGFR. N. Engl. J. Med. 362, 2380-2388 (2010).

21. Mitsudomi T, Morita S, Yatabe Y et al. Gefitinib versus cisplatin plus docetaxel in patients with non-small-cell lung cancer harbouring mutations of the epidermal growth factor receptor (WJTOG3405): an open label, randomised Phase III trial. Lancet Oncol. 11, 121-128 (2010).

22. Sequist LV, Yang JC, Yamamoto N et al. Phase III study of afatinib or cisplatin plus pemetrexed in patients with metastatic lung adenocarcinoma with EGFR mutations. J. Clin. Oncol. 31, 3327-3334 (2013). 
23. Wu YL, Zhou C, Hu CP et al. Afatinib versus cisplatin plus gemcitabine for first-line treatment of Asian patients with advanced non-small-cell lung cancer harbouring EGFR mutations (LUX-Lung 6): an open-label, randomised Phase III trial. Lancet Oncol 15, 213-222 (2014).

24. Shi YK, Wang L, Han BH et al. First-line icotinib versus cisplatin/pemetrexed plus pemetrexed maintenance therapy for patients with advanced EGFR mutation-positive lung adenocarcinoma (CONVINCE): a phase 3, open-label, randomized study. Ann. Oncol. 28, 2443-2450 (2017).

25. Park K, Tan EH, O’Byrne $\mathrm{K}$ et al. Afatinib versus gefitinib as first-line treatment of patients with EGFR mutation-positive non-small-cell lung cancer (LUX-Lung 7): a phase 2B, open-label, randomised controlled trial. Lancet Oncol. 17, 577-589 (2016).

- Phase IIb trial demonstrates that afatinib significantly improves clinical outcomes versus gefitinib in patients with EGFR mutation-positive non-small-cell lung carcinoma (NSCLC).

26. Soria JC, Ohe Y, Vansteenkiste J et al. Osimertinib in untreated EGFR-mutated advanced non-small-cell lung cancer. N. Engl. J. Med. 378, 113-125 (2018)

27. Wu YL, Cheng Y, Zhou X et al. Dacomitinib versus gefitinib as first-line treatment for patients with EGFR-mutation-positive non-small-cell lung cancer (ARCHER 1050): a randomised, open-label, phase 3 trial. Lancet Oncol. 18, 1454-1466 (2017).

28. Mok TS, Cheng Y, Zhou X et al. Improvement in overall survival in a randomized study that compared dacomitinib with gefitinib in patients with advanced non-small-cell lung cancer and EGFR-activating mutations. J. Clin. Oncol. 36, 2244-2250 (2018).

29. Ramalingam SS, Vansteenkiste J, Planchard D et al. Overall survival with osimertinib in untreated, EGFR-mutated advanced NSCLC. N. Engl. J. Med. 382, 41-50 (2020).

30. Paz-Ares L, Tan EH, O'Byrne $\mathrm{K}$ et al. Afatinib versus gefitinib in patients with EGFR mutation-positive advanced non-small-cell lung cancer: overall survival data from the phase IIb LUX-Lung 7 trial. Ann. Oncol. 28, 270-277 (2017).

31. Wu YL, Xu CR, Hu CP et al. Afatinib versus gemcitabine/cisplatin for first-line treatment of Chinese patients with advanced non-small-cell lung cancer harboring EGFR mutations: subgroup analysis of the LUX-Lung 6 trial. Onco. Targets Ther. 11, 8575-8587 (2018).

-• Subanalysis of the Phase III LUX-Lung 6 trial that assesses the efficacy and safety of afatinib versus chemotherapy specifically in Chinese patients.

32. Yang JC, Wu YL, Schuler M et al. Afatinib versus cisplatin-based chemotherapy for EGFR mutation-positive lung adenocarcinoma (LUX-Lung 3 and LUX-Lung 6): analysis of overall survival data from two randomised, phase 3 trials. Lancet Oncol. 16, 141-151 (2015).

- Overall survival (OS) analysis of LUX-Lung 6 and LUX-Lung 3 demonstrates that afatinib significantly improves OS versus chemotherapy in patients with tumors harboring an EGFR Del19 mutation.

33. Wu Y, Tu H, Feng J et al. P2.01-99 A phase IIIb open-label study of afatinib in EGFR TKI-naïve patients with EGFR mutation-positive NSCLC: final analysis. J. Thorac. Oncol. 14, S679-S680 (2019).

34. Goto Y, Tanai C, Yoh K et al. Continuing EGFR-TKI beyond radiological progression in patients with advanced or recurrent, EGFR mutation-positive non-small-cell lung cancer: an observational study. ESMO Open 2, e000214 (2017).

35. Yap TA, Macklin-Doherty A, Popat S. Continuing EGFR inhibition beyond progression in advanced non-small cell lung cancer. Eur. J. Cancer 70, 12-21 (2017).

36. Wang S, Xing P, Yang K et al. Efficacy and safety of afatinib in a Chinese population with advanced lung adenocarcinoma with sensitive EGFR mutations. Thorac. Cancer 10, 1461-1468 (2019).

- A 'real-world' analysis of outcomes in Chinese patients with EGFR mutation-positive NSCLC treated with afatinib.

37. Lin YT, Chen JS, Liao WY et al. Clinical outcomes and secondary epidermal growth factor receptor (EGFR) T790M mutation among first-line gefitinib, erlotinib and afatinib-treated non-small cell lung cancer patients with activating EGFR mutations. Int. J. Cancer 144, 2887-2896 (2019).

38. Kuan FC, Li SH, Wang CL et al. Analysis of progression-free survival of first-line tyrosine kinase inhibitors in patients with non-small cell lung cancer harboring leu858Arg or exon 19 deletions. Oncotarget 8, 1343-1353 (2017).

39. Tu CY, Chen CM, Liao WC et al. Comparison of the effects of the three major tyrosine kinase inhibitors as first-line therapy for non-small-cell lung cancer harboring epidermal growth factor receptor mutations. Oncotarget 9, 24237-24247 (2018).

40. Liang SK, Hsieh MS, Lee MR et al. Real-world experience of afatinib as a first-line therapy for advanced EGFR mutation-positive lung adenocarcinoma. Oncotarget 8, 90430-90443 (2017).

41. Shen YC, Tseng GC, Tu CY et al. Comparing the effects of afatinib with gefitinib or Erlotinib in patients with advanced-stage lung adenocarcinoma harboring non-classical epidermal growth factor receptor mutations. Lung Cancer 110, 56-62 (2017).

42. Schuler M, Paz-Ares L, Sequist LV et al. First-line afatinib for advanced EGFRm+ NSCLC: analysis of long-term responders in the LUX-Lung 3, 6, and 7 trials. Lung Cancer 133, 10-19 (2019).

43. Schuler M, Wu YL, Hirsh V et al. First-line afatinib versus chemotherapy in patients with non-small cell lung cancer and common epidermal growth factor receptor gene mutations and brain metastases. J. Thorac. Oncol. 11, 380-390 (2016).

44. Wu Y, Tu H, Feng J et al. P1.01-98 A phase IIIb trial of afatinib in EGFRm + NSCLC: analyses of outcomes in patients with brain metastases or dose reductions. J. Thorac. Oncol. 13, S501 (2018). 
45. Kim Y, Sun J, Park K et al. P3.01-023 First-line afatinib for non-small cell lung cancer in real world practice. J. Thorac. Oncol. 12, S2209 (2017).

46. Li SH, Liu CY, Hsu PC et al. Response to afatinib in treatment-naive patients with advanced mutant epidermal growth factor receptor lung adenocarcinoma with brain metastases. Expert Rev. Anticancer Ther. 18, 81-89 (2018).

47. Melosky B, Hirsh V. Management of common toxicities in metastatic NSCLC related to anti-lung cancer therapies with EGFR-TKIs. Front. Oncol. 4, 238 (2014).

48. Edwards RL, Andan C, Lalla RV et al. Afatinib therapy: practical management of adverse events with an oral agent for non-small cell lung cancer treatment. Clin. J. Oncol. Nurs. 22, 542-548 (2018).

49. Wu Y, Tu H, Feng J et al. P1.01-98 A Phase IIIb trial of afatinib in EGFRm + NSCLC: analyses of outcomes in patients with brain metastases or dose reductions. J. Thorac. Oncol. 13, S501 (2018).

50. Yang JC, Sequist LV, Zhou C et al. Effect of dose adjustment on the safety and efficacy of afatinib for EGFR mutation-positive lung adenocarcinoma: post hoc analyses of the randomized LUX-Lung 3 and 6 trials. Ann. Oncol. 27, 2103-2110 (2016).

- Analysis of the impact of tolerability-guided dose reduction on the efficacy and safety of afatinib in the LUX-Lung 3 and 6 studies.

51. Schuler M, Tan EH, O’Byrne K et al. First-line afatinib vs gefitinib for patients with EGFR mutation-positive NSCLC (LUX-Lung 7): impact of afatinib dose adjustment and analysis of mode of initial progression for patients who continued treatment beyond progression. J. Cancer Res. Clin. Oncol. 145, 1569-1579 (2019).

52. Halmos B, Tan EH, Soo RA et al. Impact of afatinib dose modification on safety and effectiveness in patients with EGFR mutation-positive advanced NSCLC: results from a global real-world study (RealGiDo). Lung Cancer 127, 103-111 (2019).

53. Wind S, Schnell D, Ebner T et al. Clinical pharmacokinetics and pharmacodynamics of afatinib. Clin. Pharmacokinet. 56, 235-250 (2017).

54. Geater SL, Xu CR, Zhou C et al. Symptom and quality of life improvement in LUX-Lung 6: an open-label phase III study of afatinib versus cisplatin/gemcitabine in Asian patients with EGFR mutation-positive advanced non-small-cell lung cancer. J. Thorac. Oncol. 10, 883-889 (2015).

55. Wu YL, Hirsh V, Sequist LV et al. Does EGFR mutation type influence patient-reported outcomes in patients with advanced EGFR mutation-positive non-small-cell lung cancer? Analysis of two large, phase III studies comparing afatinib with chemotherapy (LUX-Lung 3 and LUX-Lung 6). Patient 11, 131-141 (2018).

56. Hirsh V, Yang JCH, Tan EH et al. First-line afatinib (A) vs gefitinib (G) for patients (pts) with EGFR mutation positive (EGFRm+) NSCLC (LUX-Lung 7): patient-reported outcomes (PROs) and impact of dose modifications on efficacy and adverse events (AEs). Presented at the 2016 annual meeting of the American Society of Clinical Oncology. J. Clin. Oncol. 34, 9046-9046; Abstract 9046 (2016).

57. Sequist LV, Waltman BA, Dias-Santagata D et al. Genotypic and histological evolution of lung cancers acquiring resistance to EGFR inhibitors. Sci. Transl. Med. 3, 75ra26 (2011).

58. Yang JC, Ahn MJ, Kim DW et al. Osimertinib in pretreated T790M-positive advanced non-small-cell lung cancer: AURA study phase II extension component. J. Clin. Oncol. 35, 1288-1296 (2017).

59. Arcila ME, Oxnard GR, Nafa K et al. Rebiopsy of lung cancer patients with acquired resistance to EGFR inhibitors and enhanced detection of the T790M mutation using a locked nucleic acid-based assay. Clin. Cancer Res. 17, 1169-1180 (2011).

60. Jenkins S, Yang JC, Janne PA et al. EGFR mutation analysis for prospective patient selection in two phase II registration studies of osimertinib. J. Thorac. Oncol. 12, 1247-1256 (2017).

61. Hochmair MJ, Buder A, Schwab S et al. Liquid-biopsy-based identification of EGFR T790M mutation-mediated resistance to afatinib treatment in patients with advanced EGFR mutation-positive NSCLC, and subsequent response to osimertinib. Target. Oncol. 14, 75-83 (2019).

62. Wu SG, Liu YN, Tsai MF et al. The mechanism of acquired resistance to irreversible EGFR tyrosine kinase inhibitor-afatinib in lung adenocarcinoma patients. Oncotarget 7, 12404-12413 (2016).

63. Tanaka K, Nosaki K, Otsubo K et al. Acquisition of the T790M resistance mutation during afatinib treatment in EGFR tyrosine kinase inhibitor-naïve patients with non-small cell lung cancer harboring EGFR mutations. Oncotarget 8, 68123-68130 (2017).

64. Nakamura T, Nakashima C, Komiya K et al. Mechanisms of acquired resistance to afatinib clarified with liquid biopsy. PLoS ONE 13, e0209384 (2018).

65. Zhong WZ, Zhou Q, Wu YL. The resistance mechanisms and treatment strategies for EGFR-mutant advanced non-small-cell lung cancer. Oncotarget 8, 71358-71370 (2017).

66. Westover D, Zugazagoitia J, Cho BC et al. Mechanisms of acquired resistance to first- and second-generation EGFR tyrosine kinase inhibitors. Ann. Oncol. 29, i10-i19 (2018).

67. Duan J, Wang S, Wang Z et al. P2.01-101 Dynamic monitoring of gene alterations with ctDNA by NGS for EGFR mutated lung adenocarcinoma treated with gefitinib in BENEFIT study (CTONG 1405). J. Thorac. Oncol. 13, S704 (2018). 
68. Wang Z, Cheng Y, An T et al. Detection of EGFR mutations in plasma circulating tumour DNA as a selection criterion for first-line gefitinib treatment in patients with advanced lung adenocarcinoma (BENEFIT): a phase 2, single-arm, multicentre clinical trial. Lancet Respir. Med. 6, 681-690 (2018).

69. Blakely CM, Watkins TBK, Wu W et al. Evolution and clinical impact of co-occurring genetic alterations in advanced-stage EGFR-mutant lung cancers. Nat. Genet. 49, 1693-1704 (2017).

70. Mok TS, Wu YL, Ahn MJ et al. Osimertinib or platinum-pemetrexed in EGFR T790M-positive lung cancer. N. Engl. J. Med. 376, 629-640 (2017).

71. Hochmair MJ, Morabito A, Hao D et al. Sequential treatment with afatinib and osimertinib in patients with EGFR mutation-positive non-small-cell lung cancer: an observational study. Future Oncol. 14, 2861-2874 (2018).

-• Analysis of the observational GioTag study that assesses clinical outcomes in patients with EGFR mutation-positive NSCLC treated with sequential afatinib and osimertinib.

72. Hochmair MJ, Morabito A, Hao D et al. Sequential afatinib and osimertinib in patients with EGFR mutation-positive non-small-cell lung cancer: updated analysis of the observational GioTag study. Future Oncol. 15, 2905-2914 (2019).

-. Updated analysis of the observational GioTag study including analysis of OS.

73. Park K, Bennouna J, Boyer $\mathrm{M}$ et al. Sequencing of therapy following first-line afatinib in patients with EGFR mutation-positive non-small cell lung cancer. Lung Cancer 132, 126-131 (2019).

74. Tamiya M, Tamiya A, Suzuki H et al. Which is better EGFR-TKI followed by osimertinib: afatinib or gefitinib/erlotinib? Anticancer Res. 39, 3923-3929 (2019).

75. Reck M, Mok TSK, Nishio M et al. Atezolizumab plus bevacizumab and chemotherapy in non-small-cell lung cancer (IMpower150): key subgroup analyses of patients with EGFR mutations or baseline liver metastases in a randomised, open-label phase 3 trial. Lancet Respir. Med. 7, 387-401 (2019).

76. Liao BC, Griesing S, Yang JC. Second-line treatment of EGFR T790M-negative non-small cell lung cancer patients. Ther. Adv. Med. Oncol. 11, 1758835919890286 (2019).

77. Haura EB, Cho BC, Lee JS et al. JNJ-61186372 (JNJ-372), an EGFRcMet bispecific antibody, in EGFR-driven advanced non-small cell lung cancer (NSCLC). J. Clin. Oncol. 37, 9009-9009 (2019).

78. Oxnard GR, Yang JC, Yu H et al. TATTON: a multi-arm, phase Ib trial of osimertinib combined with selumetinib, savolitinib, or durvalumab in EGFR-mutant lung cancer. Ann. Oncol. 31, 507-516 (2020).

79. Kao HF, Hong RL. Pembrolizumab and afatinib for recurrent or metastatic head and neck squamous cell carcinoma. Ann. Oncol. 29, viii379 (2018).

80. Schoenfeld AJ, Arbour KC, Rizvi $\mathrm{H}$ et al. Severe immune-related adverse events are common with sequential PD-(L) 1 blockade and osimertinib. Ann. Oncol. 30, 839-844 (2019).

81. Yang JJ, Zhou Q, Yan HH et al. A phase III randomised controlled trial of erlotinib vs gefitinib in advanced non-small cell lung cancer with EGFR mutations. Br. J. Cancer 116, 568-574 (2017).

82. Cho BC, Chewaskulyong B, Lee KH et al. Osimertinib versus standard of care EGFR TKI as first-line treatment in patients with EGFRm advanced NSCLC: FLAURA Asian subset. J. Thorac. Oncol. 14, 99-106 (2019).

83. Zhou C, Cheng Y, He Y et al. Osimertinib vs standard of care (SoC) EGFR-TKI as first-line treatment in Chinese patients with EGFRm advanced NSCLC. J. Thorac. Oncol. 13,S507-S508 (2018).

84. Nakagawa K, Garon EB, Seto T et al. RELAY: a multinational, double-blind, randomized Phase III study of erlotinib (ERL) in combination with ramucirumab (RAM) or placebo $(\mathrm{PL})$ in previously untreated patients with epidermal growth factor receptor mutation-positive (EGFRm) metastatic non-small cell lung cancer (NSCLC). J. Clin. Oncol. 37(Suppl.), 9000(2019).

85. Noronha V, Joshi A, Maruti Patil V et al. Gefitinib versus gefitinib plus pemetrexed and carboplatin chemotherapy in EGFR-mutated lung cancer. J. Clin. Oncol. 38, 124-136 (2020).

86. Hosomi Y, Morita S, Sugawara $S$ et al. Gefitinib alone versus gefitinib plus chemotherapy for non-small-cell lung cancer with mutated epidermal growth factor receptor: NEJ009 study. J. Clin. Oncol. 38, 115-123 (2019).

87. Saito H, Fukuhara T, Furuya N et al. Erlotinib plus bevacizumab versus erlotinib alone in patients with EGFR-positive advanced non-squamous non-small-cell lung cancer (NEJ026): interim analysis of an open-label, randomised, multicentre, phase 3 trial. Lancet Oncol. 20, 625-635 (2019).

88. Ramalingam SS, Cheng Y, Zhou C et al. Mechanisms of acquired resistance to first-line osimertinib: preliminary data from the phase III FLAURA study. Presented at the 2018 European Society of Medical Oncology (ESMO) Congress. Ann. Oncol. 29, viii740; Abstract LBA50 (2018).

89. Remon J, Menis J, Hasan B et al. The APPLE trial: feasibility and activity of AZD9291 (osimertinib) treatment on positive plasma T790M in EGFR-mutant NSCLC patients. EORTC 1613. Clin. Lung Cancer 18, 583-588 (2017). 


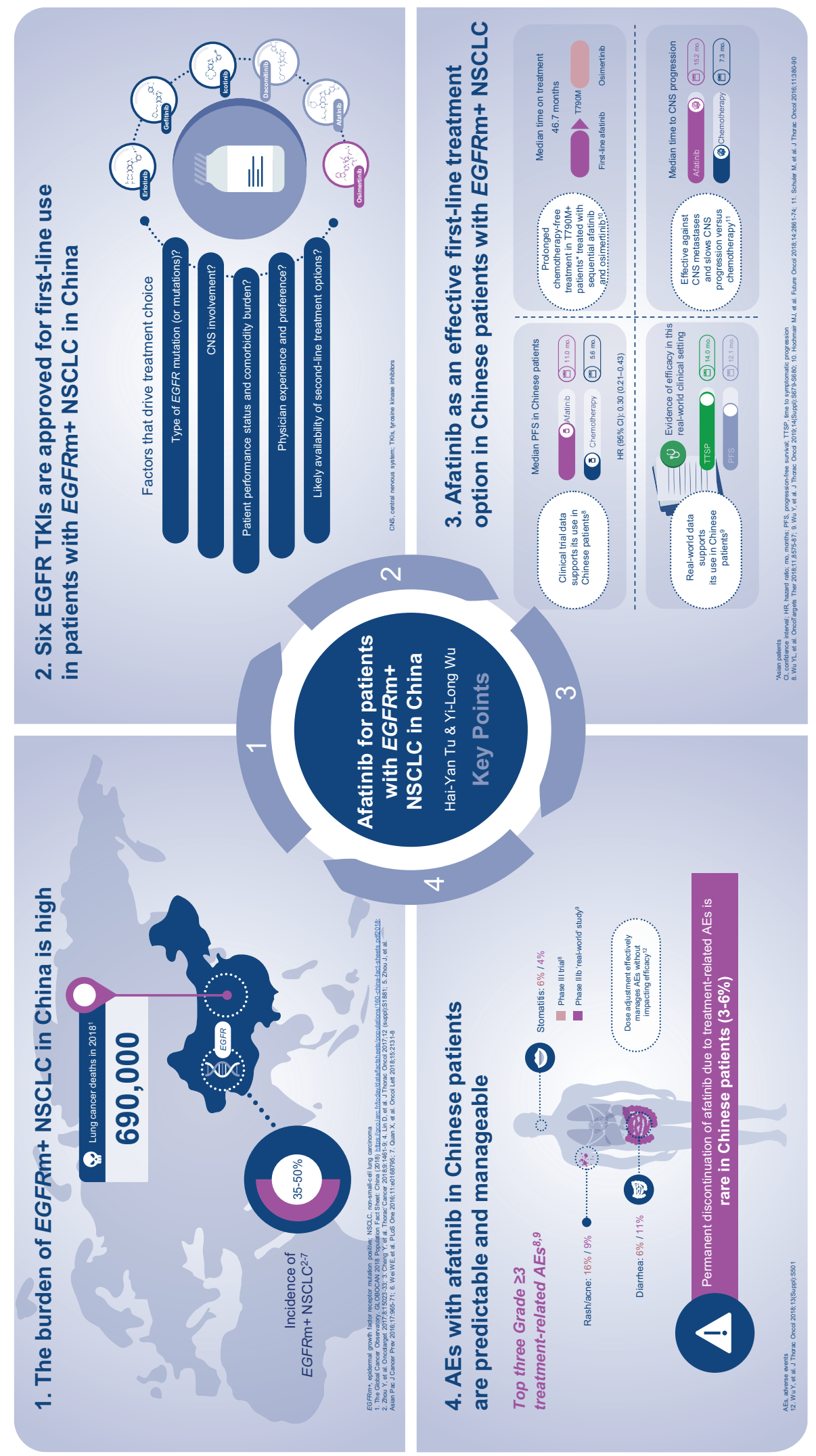

